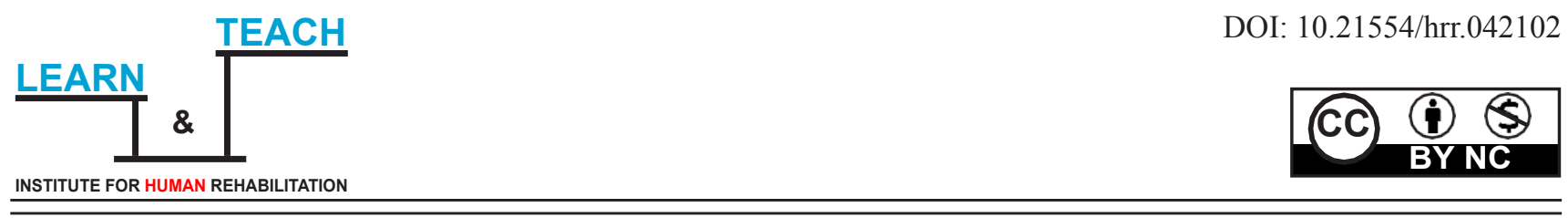

\title{
A PROSPECTIVE EVALUATION OF ORTHOTIC DEVICE IMPLICATION ON THE LOWER LIMB IN CHILDREN WITH CEREBRAL PALSY
}

\author{
Akshay Kumar', Vinita ${ }^{2}$ \\ ${ }^{\text {I}}$ Prosthetist \& Orthotist, Department of Prosthetics \& Orthotics, Composite Regional Centre for Skill Development, \\ Rehabilitation and Empowerment of Persons With Disabilities, Kozhikode, Kerala, India. \\ ${ }^{2}$ Prosthetist \& Orthotist, Pace Rehabilitation, Research, and Artificial Limb Centre, Kozhikode, Kerala.
}

Original scientific paper

Received: 2020/11/17

Accepted: 2021/2/16

\begin{abstract}
$C P$ is the foremost cause of motor disability in children and affects two to five children in 1000 live births worldwide. It is been recognized as a global concern medically, socially, and economically due to the increasing financial burden to the states. About half of the children with cerebral palsy suffer a range of motion difficulties. The study aims to understand the role of orthotic devices and its efficacy in the treatment of the patient with cerebral palsy and gait improvement. An online database of Pubmed, Science Direct, Google Scholar, etc. searched to find the articles with the keywords, cerebral palsy, orthotic devices, orthosis, lower limb, and results were synthesized and narrated to explore the effectiveness of the devices on the gait pattern and quality of life of a patient with cerebral palsy. The database search was done without the barrier of dates and regions. Orthoses applied externally appear to be supported and had a diverse effect on gait depending on the type of orthosis. However, the relationship between gait pattern and AFOs and ankle properties is yet to be established. Additional research in this area is needed to complement the development of passive and active AFOs to provide larger improvements in walking capabilities.
\end{abstract}

Keywords: cerebral palsy, orthotic devices, orthosis, lower limb

\section{INTRODUCTION}

Cerebral palsy is a neurological condition following brain injury that occurs before the complete development of cerebral as brain development occurs during the first two years of life. It can occur during prenatal, perinatal, or postnatal periods (Bass, 1999; Krigger, 2006). It is non-progressive motor disorders of movement and posture, which is heterogeneous and permanent (Olama et al., 2013). In conjunction with other treatments like orthopedic and neurosurgery, therapy, baclofen, and botulinum toxin type A, an orthosis is used to prevent or assist joint movement or restrict contracture (Morris, 2007). CP is the foremost cause of motor disability in children and affects two to five children in 1000 live births worldwide. It is been recognized as a global concern medically, socially, and economically due to the increasing financial burden to the states (Lee et al., 2014). About half of the children with a cerebral palsy suffer a range of motion difficulties and later on, this impairment limits the movement in cerebral palsy patients causing deformed joints and gait pattern deviations (Maas et al., 2012).

\footnotetext{
Correspondence to:

Kozhikode, Kerala, India.

Phone: 918434164590.

E-mail: akshaypoist@gmail.com;
}

Akshay Kumar, Prosthetist \& Orthotist, Department of Prosthetics \& Orthotics, Composite Regional Centre for Skill Development, Rehabilitation and Empowerment of Persons With Disabilities 
The importance of orthoses is important in pediatrics to implicate it as a conservative treatment method due to the flexible nature of the condition. However, it can be applied in all age groups (Schwarze et al., 2019).

\section{Hypothesis}

The hypothesis is children with cerebral palsy using orthosis like AFO, KAFO, FRO, etc for their lower limb may help in minimizing complications like contracture, improves gait pattern, postural stability, and quality of life in contrast to children left untreated. This study was done to understand the role of orthotic devices and its efficacy in the treatment of the patient with cerebral palsy and gait improvement through research literature available online. it also tries to find the future direction of the implementation of the orthotic devices for the same.

\section{METHOD AND MATERIALS}

A thorough search of major databases published in English is done without restriction on dates and regions. The major database searched were PubMed, Science Direct, Google scholar. Apart from that other search engine as BioMed Central, Cochrane Library, Wiley online library, Sage journals, PLOS ONE. The search was carried out using keywords cerebral palsy, orthotic devices, lower limb, and Mesh words of the relevant.1699 articles found using the relevant words containing research articles, review articles, encyclopedia, and book chapters. A total of 97 articles scrutinized for the review based on inclusion criteria and 44 articles meet the study purpose.

The articles included in the study have targeted the outcome of the various lower limb orthosis like AFOs, FROs, etc. on children with cerebral palsy. Articles with various study designs are selected for the study and analysis to find the effectiveness of the result.

\section{Critical Analysis:}

In a single randomized controlled study performed on 66 children with cerebral palsy to measure the ankle dorsiflexion at full knee extension, ankle, and knee flexion during gait and gross motor function. Participants were divided into three groups each of 22 participants.where as the first two groups were treated with orthosis to prevent a decrease in ankle ROM for one year and the third group acted as a controlled group given therapies and manual stretching. The results of this study indicated a positive treatment approach for children with cerebral palsy and advocate further studies to emulate the effects on gait patterns (Maas et al., 2012).

Twister Wrap Orthosis (TWO) could be effective in foot pressure distribution and postural balance in children with spastic diplegic cerebral palsy. An effectiveness measure on the Biodex stability system pre and post 12 weeks done. Thirty children of ages ranging from 6 to 8 participated in the study. all were the case of spastic diplegic cerebral palsy and inclusion criteria were similar.
The study group consisted of fifteen children went through conventional therapy program, AFO implication, and Twister Wrap Orthosis (TwO). Whereas the control group was given only therapy and AFO consisting of the same number of children (Eid et al., 2018).

A hinged AFO with 10 degrees of dorsiflexion would have a positive effect on the gait pattern of a cerebral palsy child preferably bearing a barefoot and general hinged AFO by improving cadence, step length, stride length, and gait velocity. GAITRite ${ }^{\circledR}$ was used for the gait analysis in conditions of barefoot, wearing regular hinged AFO and hinged AFO with 10-degree dorsiflexion. The child undergone the study was diagnosed with spastic diplegia but ambulated independently with the use of hinged AFO (Son et al., 2019). The hinged AFOs shortened the duration of Sitting to Standing (STS) by increasing initial knee flexion and the final angle of dorsiflexion contrast to barefoot (Park et al., 2004a).

In support of that hinged AFOs may help in improving the postural control mechanism. Nevertheless, postural stability in side-by-side needs further studies to establish the result (Rha et al., 2010). and AFOs and hinged AFOs can be helpful in ambulation for children with cerebral palsy having dynamic equinus deformities and reducing energy expenditure (Balaban et al., 2007; Chen et al., 2017; Dursun et al., 2002). Also, a cross-sectional study on 2200 children with CP (Cerebral Palsy) confirms that over 73 percent of children with CP (Cerebral Palsy)achieved their functional goals, and maintenance or improvement in ankle-dorsiflexion using AFOs (Wingstrand et al., 2014). However, randomized cross-over Wilcoxon signed-rank test study results on 11 children with $\mathrm{CP}$ suggests larger studies. The results of this study were not consistently enhanced the walking activity (Bjornson et al., 2016).

Walking with tuned AFO-FC in contrast to barefoot and non-tuned results in efficient gait parameters in several key points (Abd El-Kafy, 2014; Eddison et al., 2020). A quasi-experimental design study results on 30 spastic diplegic CP concluded that hinged AFOs gives better result in improving gross motor function than solid AFOs on standing and walking (Dalvand et al., 2013). and FRAFOS (Floor Reaction Ankle Foot Orthosis) may upgrade the knee alignment, but postural control may not improve (Bahramizadeh et al., 2012).

A case study utilizing quantitative biomechanical techniques favors the more natural ankle motion and better lower limb symmetry, and reduced knee moments during stance while using a hinged AFO contrast to solid AFO.WATSMART video system and Kistler plate were used to collect kinematic coordinate data and ground reaction force data respectively (Middleton et al., 1988). Night orthosis is also assumed to be vital in providing postural control in children with CP (Mol et al., 2012).

The pre-post experimental study design included 32 children with (Spastic Cerebral Palsy) SCP to evaluate 
the effects of optimized FROs for ankle stiffness. a homogenous group of patients walking with excessive knee flexion in midstance were studied on the walking energy cost in children with (Spastic Cerebral Palsy) $\mathrm{SCP}$, in contrast to walking with shoes alone. The FROs resulted in the reduction of knee flexion during single stance phase and will help to identify predictors of treatment success (Kerkum et al., 2013a). The best outcome of knee extension and sagittal plane knee extensor momentum can be achieved in midstance using Floor reaction ankle-foot orthosis (Rogozinski et al., 2009a).

Three-dimensional kinematic data were collected through ANOVAs to measure dependent measures of 21 children with spastic diplegia walked with the bilateral orthosis. Eleven patients used solid orthosis and ten used articulated orthosis. However, the results show an increase in stride length, energy recovery, and potential energy and kinetic energy for both groups. and AFOS can reduce work to walk (Bennett et al., 2012a). Nevertheless, step length shown improvements along with speed and ankle function for the average AFO users. this data extracted from 378 individuals with 601 visits using statistical analysis consisted of paired $t$-tests and multivariate analysis of variance scores (Ries et al., 2015). Foot orthosis acts as a medical device and minimizes the pathologies loading on the structural components of the foot to allow more natural motion and balance and postural controls (Olama et al., 2013).

To measure gait quality analysis Edinburgh Visual Gait Score (EVGS) is used as an authenticated observational gait analysis scale. In ambulatory children with cerebral palsy Edinburgh Visual Gait Score (EVGS) used for gait analysis evaluation. A cross-sectional cohort study was conducted using EVGS to determine the effects of AFOs and sensomotoric orthoses (SMotOs) in children with Cerebral palsy gait patterns. All the included participants (eleven) were using SMotOs and AFOs. The results showed improved gait with SMotOs and it can be an appropriate option in clinical settings for this population. However, further study is needed (MacFarlane et al., 2020).

A study performed with ventral shell spring-hinged AFO (vAFO) to know the implication effect of varying AFO stiffness on individual gait biomechanics and efficiency in cerebral palsy. a 3D-gait analysis and a 6-minute walk test on Fifteen children who walk with couch gait/excessive knee flexion in the stance phase investigated. all vAFOs improved the knee angle, net moment, and decreased the net energy cost contrast to shoes-only (Kerkum et al., 2015). Also, the data proves that optimized vAFOs reduce knee flexion in stance and enhance gait in CP children (Kerkum et al., 2016).

\section{RESULTS AND DISCUSSION}

Orthoses applied externally appear to be supported and had a diverse effect on gait depending on the type of orthosis (Lampe et al., 2004). However, the older age of users got less effective development of skill and only young children benefited more (Bjornson et al., 2006a).

About $90 \%$ of children with Cerebral Palsy had imbalance problems during walking and 54\% were unable to walk without assistance. So, ambulation disorder is an essential limiting factor in children with Spastic diplegic Cerebral Palsy (Kadhim \& Miller, 2014). The first study to find the effect and efficacy of individually optimized FRO on a homogenous group, together with effects evaluation on multiple ICF levels may result in optimal FRO treatment in the defined patient group (Kerkum et al., 2013b).

It is recommended that AFOs can help in improving the characteristics of gait in an individual patient and this finding is not new. However, the relationship between gait pattern and AFOs and ankle properties is yet to be established and focus needs to be paid to the individual gait characteristics and the features of AFO would be beneficial (Bennett et al., 2012b).

Gait analysis using tuned AFO-FC produced improvement in hip flexion and extension, posterior pelvic tilt, and knee extension (Eddison et al., 2020). However, there was no pelvic change in pelvic tilt and hip flexion observed using the hinged AFOs (Park et al., 2004b). and such instrumented protocol may use as a quantitative tool to support and reveal an appropriate line of orthotic treatment (Contini et al., 2019). So, the dysfunction or foot deformities either maintained or improve using long-term different kinds of AFOs (Liu et al., 2018).

In inhibiting spastic activation, hinged ankle-foot Orhtosis /Orthotic management may be an effective management technique in cerebral palsy patients (Lindskov et al., 2020). However, The ideal knee and hip flexion contracture of less than or equal to 10 to 15 degrees were found to the limit of efficacy using Floor reaction Ankle-Foot Orthosis (Rogozinski et al., 2009b).

Floor Reaction Ankle Foot Orthosis (FRAFO) was also effective to improve knee and ankle extension in stance (Lucareli et al., 2007). The FES (Functional Electrical Stimulation) application to Children with Cerebral Palsy produced evidence of muscle plasticity in the tibialis anterior (TA) anatomical cross-sectional area and muscle thickness increased. However, permanent improvement in voluntary ankle control was not evidenced (Damiano et al., 2013).

\section{CONCLUSION}

Different kinds of AFOs improved gait patterns in children with cerebral palsy in contrast to barefoot walking resulted in efficient progression of the foot in stance and restricts premature movement (Abel et al., 1998). All the studies performed could fail to deliver knowledge about the effect of materials and orthosis design. Hence, that needed to be conduct research in such a direction. There still a need for more research on kinematic effects of using various kinds of AFOs and devices (Eddison et al., 2020). 
Further research needs to understand the long-term effect of orthoses on gait patterns and gross motor skill development (Bjornson et al., 2006b). Detailed information on the design features of AFOs according to the severity and limb impairment may be the future aspect in children with cerebral palsy (Chisholm and Perry, 2012). Additional research in this area is needed to complement the development of passive and active AFOs to provide larger improvements in walking capabilities.

\section{ACKNOWLEDGMENT}

The author(s) are grateful to Dr.Roshan Bijlee $\mathrm{KN}$, the director of Composite Regional Center for Persons with disabilities, Kozhikode, Kerala for his constant technical support.

\section{REFERENCES}

Abd El-Kafy, E.M., 2014. The clinical impact of orthotic correction of lower limb rotational deformities in children with cerebral palsy: A randomized controlled trial. Clinical Rehabilitation, 28, 1004-1014. https://doi. org/10.1177/0269215514533710

Abel, M.F., Juhl, G.A., Vaughan, C.L., Damiano, D.L., 1998. Gait assessment of fixed ankle-foot orthoses in children with spastic diplegia. Archives of Physical Medicine and Rehabilitation, 79, 126-133. https://doi.org/10.1016/ S0003-9993(98)90288-X

Bahramizadeh, M., Mousavi, M.E., Rassafiani, M., Aminian, G., Ebrahimi, I., Karimlou, M., O'Toole, G., 2012. The effect of floor reaction ankle foot orthosis on postural control in children with spastic cerebral palsy. Prosthetics and Orthotics International, 36, 71-76. https://doi. org/10.1177/0309364611429855

Balaban, B., Yasar, E., Dal, U., Yazicioglu, K., Mohur, H., Kalyon, T.A., 2007. The effect of hinged ankle-foot orthosis on gait and energy expenditure in spastic hemiplegic cerebral palsy. Disability and Rehabilitation, 29, 139-144. https:// doi.org/10.1080/17483100600876740

Bass, N., 1999. Cerebral palsy and neurodegenerative disease. Current Opinion in Pediatrics, 11, 504-507. https://doi. org/10.1097/00008480-199912000-00005

Bennett, B.C., Russell, S.D., Abel, M.F., 2012a. The effects of ankle foot orthoses on energy recovery and work during gait in children with cerebral palsy. Clinical Biomechanics, 27, 287-291. https://doi.org/10.1016/j. clinbiomech.2011.09.005

Bennett, B.C., Russell, S.D., \& Abel, M.F. (2012b). The effects of ankle foot orthoses on energy recovery and work during gait in children with cerebral palsy. Clinical Biomechanics, 27, 287-291. https://doi.org/10.1016/j. clinbiomech.2011.09.005

Bjornson, K., Zhou, C., Fatone, S., Orendurff, M., Stevenson, R., $\&$ Rashid, S. (2016). The Effect of Ankle-Foot Orthoses on Community-Based Walking in Cerebral Palsy. Pediatric Physical Therapy, 28, 179-186. https://doi.org/10.1097/ PEP.0000000000000242

Bjornson, K.F., Schmale, G.A., Adamczyk-Foster, A., McLaughlin, J., 2006a. The Effect of Dynamic Ankle Foot Orthoses on Function in Children With Cerebral Palsy. Journal of Pediatric Orthopaedics, 26, 773-776. https:// doi.org/10.1097/01.bpo.0000242377.10596.0f

Bjornson, K.F., Schmale, G.A., Adamczyk-Foster, A. \& McLaughlin, J. (2006b). The Effect of Dynamic Ankle Foot Orthoses on Function in Children With Cerebral Palsy. Journal of Pediatric Orthopaedics, 26, 773-776. https:// doi.org/10.1097/01.bpo.0000242377.10596.0f
Chen, W., Liu, X., Pu, F., Yang, Y., Wang, L., Liu, H., \& Fan, Y. (2017). Conservative treatment for equinus deformity in children with cerebral palsy using an adjustable splintassisted ankle-foot orthosis. Medicine, 96, e8186. https:// doi.org/10.1097/MD.0000000000008186

Chisholm, A.E., \& Perry, S.D. (2012). Ankle-foot orthotic management in neuromuscular disorders: Recommendations for future research. Disability and Rehabilitation: Assistive Technology. https://doi.org/10.3109/17483107.2012.68094 0

Contini, B.G., Bergamini, E., Alvini, M., Di Stanislao, E., Di Rosa, G., Castelli, E., Vannozzi, G., \& Camomilla, V. (2019). A wearable gait analysis protocol to support the choice of the appropriate ankle-foot orthosis: A comparative assessment in children with Cerebral Palsy. Clinical Biomechanics, 70, 177-185. https://doi.org/10.1016/j. clinbiomech.2019.08.009

Dalvand, H., Dehghan, L., Feizi, A., Hosseini, S.A., \& Amirsalari, S. (2013). The impacts of hinged and solid ankle-foot orthoses on standing and walking in children with spastic diplegia. Iranian Journal of Child Neurology, 7, 12-19. https://doi.org/10.22037/ijcn.v7i4.3558

Damiano, D.L., Prosser, L.A., Curatalo, L.A., \& Alter, K.E. (2013). Muscle plasticity and ankle control after repetitive use of a functional electrical stimulation device for foot drop in cerebral palsy. Neurorehabilitation and Neural Repair, 27, 200-207. https://doi.org/10.1177/1545968312461716

Dursun, E., Dursun, N., \& Alican, D. (2002). Ankle-foot orthoses: Effect on gait in children with cerebral palsy. Disability and Rehabilitation, 24, 345-347. https://doi. org/10.1080/0963820110090724

Eddison, N., Healy, A., Needham, R., \& Chockalingam, N. (2020). The effect of tuning ankle foot orthoses-footwear combinations on gait kinematics of children with cerebral palsy: A case series. Foot, 43, 101660. https://doi. org/10.1016/j.foot.2019.101660

Eid, M.A., Aly, S.M., \& Mohamed, R.A. (2018). Effect of twister wrap orthosis on foot pressure distribution and balance in diplegic cerebral palsy. Journal of Musculoskeletal Neuronal Interactions, 18, 543-550.

Kadhim, M., \& Miller, F. (2014). Crouch gait changes after planovalgus foot deformity correction in ambulatory children with cerebral palsy. Gait and Posture, 39, 793798. https://doi.org/10.1016/j.gaitpost.2013.10.020

Kerkum, Y.L., Buizer, A.I., van den Noort, J.C., Becher, J.G., Harlaar, J., \& Brehm, M.-A. (2015). The Effects of Varying Ankle Foot Orthosis Stiffness on Gait in Children with Spastic Cerebral Palsy Who Walk with Excessive Knee Flexion. PLOS ONE, 10, e0142878. https://doi. org/10.1371/journal.pone. 0142878

Kerkum, Y.L., Harlaar, J., Buizer, A.I., Van Den Noort, J.C., Becher, J.G., \& Brehm, M.A. (2016). An individual approach for optimizing ankle-foot orthoses to improve mobility in children with spastic cerebral palsy walking with excessive knee flexion. Gait and Posture, 46, 104111. https://doi.org/10.1016/j.gaitpost.2016.03.001

Kerkum, Y.L., Harlaar, J., Buizer, A.I., Van Den Noort, J.C., Becher, J.G., \& Brehm, M.A. (2013a). Optimising Ankle Foot Orthoses for children with Cerebral Palsy walking with excessive knee flexion to improve their mobility and participation; protocol of the AFO-CP study. BMC Pediatrics, 13, 17. https://doi.org/10.1186/1471-2431-1317

Kerkum, Y.L., Harlaar, J., Buizer, A.I., Van Den Noort, J.C., Becher, J.G., \& Brehm, M.A. (2013b). Optimising Ankle Foot Orthoses for children with Cerebral Palsy walking with excessive knee flexion to improve their mobility and participation; protocol of the AFO-CP study. BMC Pediatrics, 13, 17. https://doi.org/10.1186/1471-2431-1317

Krigger, K.W. (2006). Cerebral Palsy: An Overview, American Family Physician. 
Lampe, R., Mitternacht, J., Schrödl, S., Gerdesmeyer, L., Natrath, M., \& Gradinger, R. (2004). Influence of orthopaedictechnical aid on the kinematics and kinetics of the knee joint of patients with neuro-orthopaedic diseases. Brain and Development, 26, 219-226. https://doi.org/10.1016/S03877604(03)00129-3

Lee, R.W., Poretti, A., Cohen, J.S., Levey, E., Gwynn, H., Johnston, M. V., Hoon, A.H., \& Fatemi, A. (2014). A Diagnostic Approach for Cerebral Palsy in the Genomic Era. NeuroMolecular Medicine, 16, 821-844. https://doi. org/10.1007/s12017-014-8331-9

Lindskov, L., Huse, A.B., Johansson, M., \& Nygård, S. (2020). Muscle activity in children with spastic unilateral cerebral palsy when walking with ankle-foot orthoses: an explorative study. Gait and Posture, 80, 31-36. https://doi. org/10.1016/j.gaitpost.2020.05.011

Liu, X.-C., Embrey, D., Tassone, C., Zvara, K., Brandsma, B., Lyon, R., Goodfriend, K., Tarima, S., \& Thometz, J. (2018). Long-Term Effects of Orthoses Use on the Changes of Foot and Ankle Joint Motions of Children With Spastic Cerebral Palsy. $P M \& R, \quad 10$, 269-275. https://doi.org/10.1016/j. pmrj.2017.08.438

Lucareli, P.R.G., Lima, M.D.O., Lucarelli, J.G.D.A., \& Lima, F.P.S. (2007). Changes in joint kinematics in children with cerebral palsy while walking with and without a floor reaction ankle-foot orthosis. Clinics 62, 63-68. https://doi. org $/ 10.1590 /$ S1807-59322007000100010

Maas, J.C., Dallmeijer, A.J., Huijing, P.A., Brunstrom-Hernandez, J.E., van Kampen, P.J., Jaspers, R.T., \& Becher, J.G. (2012). Splint: The efficacy of orthotic management in rest to prevent equinus in children with cerebral palsy, a randomised controlled trial. BMC Pediatrics, 12, 38. https://doi.org/10.1186/1471-2431-12-38

MacFarlane, C., Hing, W., \& Orr, R. (2020). Using the Edinburgh Visual Gait Score to Compare Ankle-Foot Orthoses, Sensorimotor Orthoses and Barefoot Gait Pattern in Children with Cerebral Palsy. Children 7, 54. https://doi. org/10.3390/children7060054

Middleton, E.A., Hurley, G.R.B., Mcilwain, J.S., (1988). The role of rigid and hinged polypropylene ankle-foot-orthoses in the management of cerebral palsy: A case study. Prosthetics and Orthotics International, 12, 129-135. https://doi. org/10.3109/03093648809079396

Mol, E.M., Monbaliu, E., Ven, M., Vergote, M., \& Prinzie, P. (2012). The use of night orthoses in cerebral palsy treatment: Sleep disturbance in children and parental burden or not? Research in Developmental Disabilities, 33, 341-349. https://doi.org/10.1016/j.ridd.2011.10.026

Morris, C. (2007). A review of the efficacy of lower-limb orthoses used for cerebral palsy. Developmental Medicine \& Child Neurology, 44, 205-211. https://doi. $\operatorname{org} / 10.1111 / \mathrm{j} .1469-8749.2002 . t b 00789 . x$
Olama, K.A., El-Din, S.M.N., \& Ibrahem, M.B. (2013). Role of three side support ankle-foot orthosis in improving the balance in children with spastic diplegic cerebral palsy. Egyptian Journal of Medical Human Genetics, 14, 77-85. https://doi.org/10.1016/j.ejmhg.2012.10.001

Park, E.S., Park, C. Il, Chang, H.J., Choi, J.E., \& Lee, D.S. (2004a). The effect of hinged ankle-foot orthoses on sit-to-stand transfer in children with spastic cerebral palsy. Archives of Physical Medicine and Rehabilitation, 85, 2053-2057. https://doi.org/10.1016/j.apmr.2004.05.008

Park, E.S., Park, C. Il, Chang, H.J., Choi, J.E., \& Lee, D.S. (2004b). The effect of hinged ankle-foot orthoses on sit-to-stand transfer in children with spastic cerebral palsy. Archives of Physical Medicine and Rehabilitation, 85, 2053-2057. https://doi.org/10.1016/j.apmr.2004.05.008

Rha, D., Kim, D.J., \& Park, E.S. (2010). Effect of Hinged AnkleFoot Orthoses on Standing Balance Control in Children with Bilateral Spastic Cerebral Palsy. Yonsei Medical Journal, 51, 746. https://doi.org/10.3349/ymj.2010.51.5.746

Ries, A.J., Novacheck, T.F., \& Schwartz, M.H. (2015). The Efficacy of Ankle-Foot Orthoses on Improving the Gait of Children With Diplegic Cerebral Palsy: A Multiple Outcome Analysis. $P M \& R$ 7, 922-929. https://doi.org/10.1016/j. pmrj.2015.03.005

Rogozinski, B.M., Davids, J.R., Davis, R.B., Jameson, G.G., \& Blackhurst, D.W. (2009a). The Efficacy of the FloorReaction Ankle-Foot Orthosis in Children with Cerebral Palsy. The Journal of Bone and Joint Surgery-American Volume 91, 2440-2447. https://doi.org/10.2106/ JBJS.H.00965

Rogozinski, B.M., Davids, J.R., Davis, R.B., Jameson, G.G., \& Blackhurst, D.W. (2009b). The Efficacy of the FloorReaction Ankle-Foot Orthosis in Children with Cerebral Palsy. The Journal of Bone and Joint Surgery-American, 91, 2440-2447. https://doi.org/10.2106/JBJS.H.00965

Schwarze, M., Horoba, L., Block, J., Putz, C., Alimusaj, M., Wolf, S.I., \& Dreher, T. (2019). Wearing Time of Ankle-Foot Orthoses with Modular Shank Supply in Cerebral Palsy: A Descriptive Analysis in a Clinically Prospective Approach. Rehabilitation Research and Practice 2019. https://doi. org $/ 10.1155 / 2019 / 2978265$

Son, I.H., Lee, D.G., Hong, S.K., Lee, K., \& Lee, G.C. (2019). Comparison of gait ability of a child with cerebral palsy according to the difference of dorsiflexion angle of hinged ankle-foot orthosis: A case report. American Journal of Case Reports 20, 1454-1459. https://doi.org/10.12659/ AJCR.916814

Wingstrand, M., Hägglund, G., \& Rodby-Bousquet, E. (2014). Ankle-foot orthoses in children with cerebral palsy: A cross sectional population based study of 2200 children. BMC Musculoskeletal Disorders 15, 327. https://doi. org/10.1186/1471-2474-15-327 